\title{
Increase in isolation of extended spectrum beta lactamase producing multidrug resistant non typhoidal Salmonellae in Pakistan
}

\author{
Kauser Jabeen*, Afia Zafar, Seema Irfan, Erum Khan, Vikram Mehraj and Rumina Hasan
}

\begin{abstract}
Background: Increasing resistance to quinolones and ceftriaxone in non typhoidal Salmonellae is a global concern. Resistance to quinolone and $3^{\text {rd }}$ generation cephalosporin amongst non typhoidal Salmonellae (NTS) from Pakistan has been reported in this study.

Methods: Retrospective analysis of laboratory data was conducted (1990-2006). NTS were isolated and identified from clinical samples using standard microbiological techniques. Antimicrobial susceptibility testing was performed by Kirby Bauer. Extended spectrum beta lactamase production (ESBL) was detected using combined disc method. Ciprofloxacin sensitivity was detected by nalidixic acid screening method. Minimum inhibitory concentration (MIC) of ciprofloxacin was determined by agar dilution method. Statistical analysis was performed using SPSS version 13.

Results: Analysis of 1967 NTS isolates showed a significant increase in ciprofloxacin resistance from 23\% in 2002 to $50.5 \%$ in 2006, with increased mean MIC values from 0.6 to $1.3 \mathrm{ug} / \mathrm{mL}$. Ceftriaxone resistant NTS also increased and ESBL production was seen in $98.7 \%$ isolates. These isolates exhibited high resistance against amoxicillin clavulanic acid (57\%), gentamicin (69\%), amikacin (44\%) and piperacillin tazobactam (30\%). No resistance to carbapenem was seen. Ceftriaxone resistance was significantly higher in children $<1$ year, in invasive isolates and in Salmonella Typhimurium.

Conclusions: Increase in quinolone and ceftriaxone NTS is a serious threat to public health requiring continuous surveillance and use of appropriate screening tests for laboratory detection.
\end{abstract}

\section{Background}

Non typhoidal salmonellae (NTS) are associated in approximately $5 \%$ of the patients with bacteremia, invasive infections and death [1]. Increased antimicrobial resistance has made empiric antibiotic of choice for these potentially fatal infections quite limited. Current literature recommends either a fluoroquinolones or a third generation cephalosporins as drug of choice; however increasing evidence of emergence of resistance against these antibiotics in a great concern $[2,3]$.

Outbreaks due to highly resistant NTS have been reported from a number of countries [3-6]. Surveillance of antimicrobial resistance amongst NTS is crucial, particularly in developing countries where facilities for culture and susceptibility testing are not widely accessible

\footnotetext{
* Correspondence: kausar.jabeen@aku.edu

1 Department of Pathology and Microbiology, Aga Khan University, Karachi, Pakistan

Full list of author information is available at the end of the article
}

and clinicians rely mainly on empirical therapy. While increased resistance in typhoidal Salmonellae has been reported from Pakistan, antimicrobial resistance data of NTS is unavailable. Resistance to quinolone and $3^{\text {rd }}$ generation cephalosporin with extended spectrum beta lactamase (ESBL) producing NTS have not been yet reported from this region. We have assessed antimicrobial susceptibility of NTS from Pakistan over a period of 17 years including analysis of resistance to quinolones and $3^{\text {rd }}$ generation cephalosporin.

\section{Methods \\ Setting}

This study was conducted from 1990-2006 at the Aga Khan University (AKU), a tertiary care hospital in Karachi, Pakistan. The hospital and laboratory are accredited with Joint commission of international accreditation (JCIA). Laboratory routinely participates in external 
quality control surveys with College of American pathologists (CAP). Clinical microbiology laboratory receives specimens across the country via satellite centers in 50 major cities and towns of Pakistan. The data presented here was not collected in a programmed survey but obtained in routine analysis of specimens submitted to the laboratory. All specimens although were processed in laboratory based in Karachi but the data represents strains prevalent across the country. This study was a retrospective analysis of the laboratory data and did not include any identifiable information from the patients therefore formal ethical committee approval was not obtained as per research guidelines of the institute.

\section{Specimen Selection}

Data including both invasive and non-invasive NTS was retrieved from a central computerized database. Duplicate specimens from same patients and samples with Salmonella enterica Typhi and Paratyphi were also excluded.

\section{Microbiological Methods}

During the study period NTS from stool and urine were identified biochemically using conventional tests [7]. Isolates from blood, tissues and other sterile body sites were identified using API 20E (Bio Merieux France). Suspected colonies were tested with Salmonella polyvalent antisera (A-I and Vi) (Difco). Serogroups were determined using the slide agglutination method. Non typeable Salmonella spp. were those isolates that have biochemical (API 20E) and serological profile suggestive of Salmonella but were not further classified into serogroups due to limited availability of specific O antisera (only A, B, C and D). Serotypes were determined by tube agglutination method against $\mathrm{H}$ antigen. If an isolate was not serotyped with the available $\mathrm{H}$ antisera only serogroups were reported.

Antimicrobial susceptibility testing was performed as per Clinical Laboratory Standards Institute (CLSI) criteria [8] against nalidixic acid $(30 \mu \mathrm{g})$ ciprofloxacin $(5 \mu \mathrm{g})$, ampicillin $(10 \mu \mathrm{g})$ ceftriaxone $(30 \mu \mathrm{g})$, chloramphenicol $(30 \mu \mathrm{g})$, and cotrimoxazole $(1.25 / 23.75 \mu \mathrm{g})$. ESBL production was detected by combined disc method (ceftriaxone alone and ceftriaxone-clavulanic acid) [8]. ESBL producing strains were further tested against amoxicillin-clavulanic acid $(20 / 10 \mu \mathrm{g})$, gentamicin $(10 \mu \mathrm{g})$, amikacin (30 $\mu \mathrm{g})$, piperacillin-tazobactam $(100 / 10 \mu \mathrm{g})$ and meropenem $(10 \mu \mathrm{g})$. Since year 2002 susceptibility against ciprofloxacin was determined by nalidixic acid screening method as recommended by Hakanen et al [9]. Minimum inhibitory concentrations against ciprofloxacin were determined using agar dilution method [8]. Isolates that were resistant to ampicillin, chloramphenicol and cotrimoxazole were considered multidrug resistant (MDR).

\section{Statistical Analysis}

Clinical data were analyzed using SPSS version 15.0 software. Comparisons were made between resistant and sensitive strains in terms of age, gender, specimen source and serogroups. Proportions were compared using the Chi-square test or Fisher's exact test, where appropriate. A p value of less than $5 \%$ was considered as statistically significant.

\section{Results}

During the study period, a total of 1967 NTS were identified. A characteristic seasonal pattern in isolation of NTS was observed with increased isolation in the months of May-August corresponding with the summer and monsoon season (data not shown). Majority of NTS isolates were from females (57.5\%). Isolation rate of NTS was higher in the age group under 5 years $(55.5 \%)$. Out of 1967 isolates majority were from stool (91\%) followed by blood (6\%). Most prevalent organisms were the Salmonella group B (659) including Salmonella enterica Typhimurium (257) followed by Salmonella group C (465), Salmonella group A (62) and Salmonella group D (48). Due to limited availability of a number of antisera the large group of non-typeable Salmonella (728) could not be speciated.

The resistance rates against nalidixic acid, ofloxacin and ceftriaxone were increased (Fig. 1). This increase was significant for nalidixic acid and ofloxacin ( $\mathrm{p}$ value for trend < 0.001). Between 1990 and 2001 in accordance with NCCLS guidelines interpretative criteria of Enterobacteriaceae was used to report susceptibility of NTS against fluoroquinolone, hence resistance was not detected. However, following the introduction of nalidixic acid screening method in 2002, $27 \%$ of the NTS isolates were noted to have reduced susceptibility to quinolones. Mean MIC values of ofloxacin in NTS also gradually increased during this period (Fig 2).

Ceftriaxone resistant NTS first observed in year 2000, increased gradually over the years. ESBL production was seen in $98.7 \%(n=78)$ of ceftriaxone resistant NTS. ESBL producing NTS isolates further showed resistance against amoxicillin clavulanic acid (57\%), gentamicin (69\%), amikacin (44\%) and piperacillin/tazobactam (30\%). Resistance to carbapenem was not observed. Ceftriaxone resistance was significantly higher ( $\mathrm{p}$ value $<0.001$ ) amongst organisms isolated from patients less than 1 year of age, in invasive isolates, in hospitalized patients and in Salmonella enterica Typhimurium. In comparison to other Salmonella spp., Salmonella enterica Typhimurium had significantly higher resistance to ciprofloxacin (35.5 vs. $19 \%$ ) and had higher MDR rate (39\% vs. $8 \%$ ).

Interestingly resistance against the first line drugs did not increase over the years. Rather resistance to chloram- 


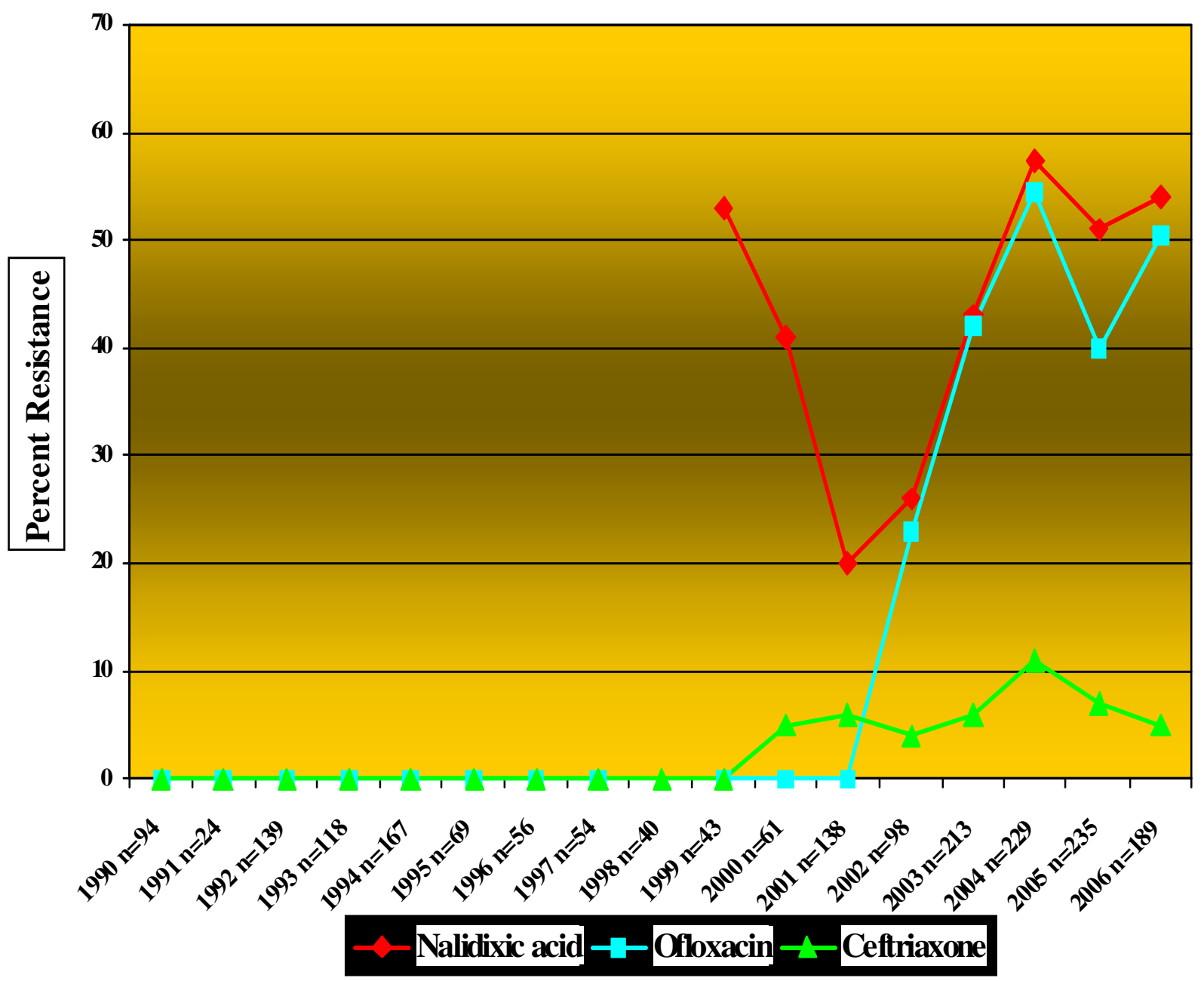

Figure 1 Showing marked increase in resistance against nalidixic acid, ofloxacin and ceftriaxone in NTS isolates at the Aga Khan University Hospital (1990-2006).

phenicol decreased from 26\% in 1990 to $7 \%$ in 2006 (p value $<0.001)$. Similarly resistance against cotrimoxazole and ampicillin remained static with minimum variations. Isolation of MDR NTS strains significantly decreased to $3 \%$ in 2006 (p value < 0.001) (Fig 3).

\section{Discussion}

Globally empirical therapy of invasive NTS infections has become challenging with the emergence of ceftriaxone and quinolone resistance. This is the first report from Pakistan reporting ESBL producing NTS isolates and increase in quinolone. We also observed recent decline in isolation of MDR NTS strains.

Since 1991 ceftriaxone resistant NTS has been reported in various studies $[6,10]$ and is reportedly mainly due to ESBL (particularly CTX-M types) and AmpC $\beta$ lactamases $[3,11]$. Within our samples, ceftriaxone resistant NTS were first isolated in the year 2000 and the rate of isolation of these strains has gradually increased over the years. $98.7 \%$ of our strains were ESBL producers and showed concurrent resistance to broad spectrum antibiotics including amoxicillin-clavulanic acid, amikacin, gentamicin and piperacillin-tazobactam. Fortunately carbapenem resistance, although reported previously in NTS was not detected in our study [12]. The significant association of these strains with patients under than 1 year of age and with invasive infections makes the management of these strains more problematic. Another concern is inappropriate detection of such resistant strains in most of the laboratories in Pakistan.

This study also reports an increase in fluoroquinolone resistance from $23 \%$ in 2002 to $50.5 \%$ in 2006 . Such an increase is in agreement with reports from other countries $[4,5]$. Prior to 2002 we were using CLSI breakpoints 


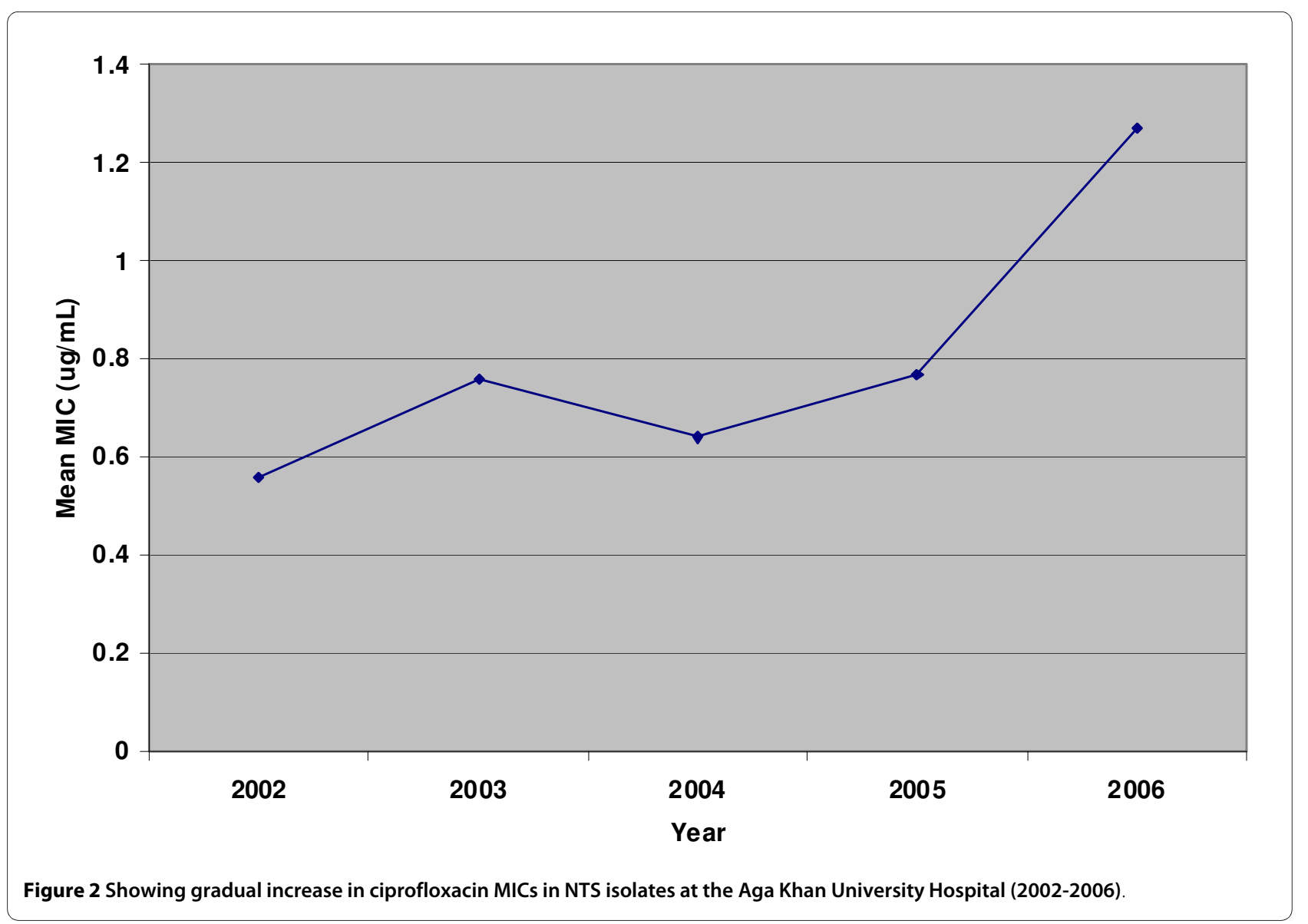

recommended at the time and were therefore not able to detect any resistance [8]. From 2002 onwards nalidixic acid screening method was employed for the detection of quinolone resistance. The rise in resistance rates correlates also with increase in mean MIC values for these strains. We recommend that accurate detection of Salmonella strains with reduced quinolone susceptibility should be reported by the clinical laboratories in Pakistan, as clinical outcome with quinolones therapy is poor.

Salmonella Typhimurium had highest resistance rates to ceftriaxone, ciprofloxacin and MDR. Increased resistance to first line drugs has been reported previously in Salmonella Typhimurium from UK and Spain [13,14]. Another study from England and Wales has also reported higher ceftriaxone resistance rates in this serotype [10]. Increased antimicrobial resistance in Salmonella Typhimurium is alarming as high mortality and invasive infections are associated with this serotype $[15,16]$.

We also observed static resistance rates to ampicillin and cotrimoxazole and a decline in resistance to chloramphenicol and MDR strains as reported previously in Kenya [17]. Another report from Pakistan reporting resistance rates in Salmonella Typhi also demonstrated declining resistance to first line drugs [18]. They corre- lated this with the overall antimicrobial consumption at population level in Karachi and showed a steady reduction in the use of the above antibiotics especially chloramphenicol. Based on their findings we can assume similar correlation in NTS as well.

\section{Conclusions}

Increased resistance to ciprofloxacin and emergence of ESBL producing NTS pose both diagnostic and management dilemma in a developing country like Pakistan. Measures should be taken to stop further emergence of resistance and their dissemination on a priority basis. Preventive strategy would require improved laboratory services, continuous surveillance and restriction of use of broad spectrum antibiotics.

\section{Funding Source}

None.

\section{Competing interests}

The authors declare that they have no competing interests.

\section{Authors' contributions}

$\mathrm{KJ}$ initiated, planned and completed the study. She also wrote the initial manuscript and SI, AZ, EK and RH critically analyzed it for intellectual content and revised it accordingly. VM performed the data analysis. All authors approved the final version. 


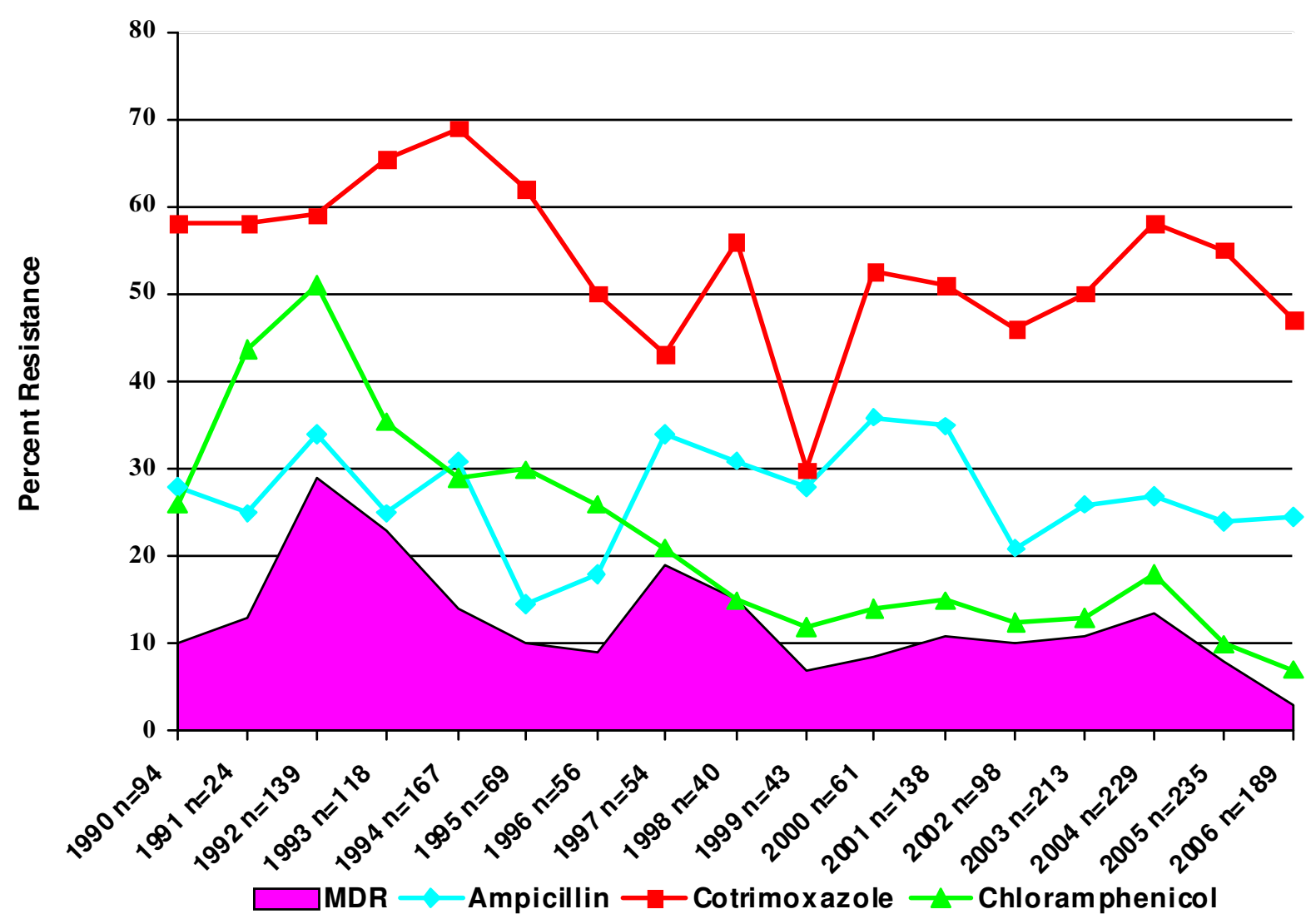

Figure 3 Showing trends of resistance against first line drugs in NTS isolates at the Aga Khan University Hospital (1990-2006)

\section{Acknowledgements}

We would also like to thank the faculty and staff of the Clinical Microbiology Laboratory Aga Khan University Hospital, Karachi for their support and help in this study.

\section{Author Details}

Department of Pathology and Microbiology, Aga Khan University, Karachi, Pakistan

Received: 31 July 2009 Accepted: 22 April 2010

Published: 22 April 2010

\section{References}

1. Mead PS, Slutsker L, Dietz V, et al:: Food related illness and death in the United States. Emerg Infect Dis 1999, 5:607-25.

2. Hohmann EL: Nontyphoidal salmonellosis. Clin Infect Dis 2001, 32:263-9.

3. Su LH, Chiu CH, Chu C, Ou JT: Antimicrobial resistance in nontyphoid Salmonella serotypes: A global challenge. Clin Infect Dis 2004, 39:546-51.

4. Murray A, Coia JE, Mather H, Brown DJ: Ciprofloxacin resistance in non typhoidal Salmonella serotypes in Scotland, 1993-2003. J Antimicrob Chemother 2005, 56:110-114

5. Molbak Kare, Baggesen DL, Aarestrup FM, Ebbesen JM, Engberg J, Frydendahl K, et al:: An outbreak of multidrug resistant, quinolone resistant Salmonella enterica Serotype Typhimurium DT104. NEJM 1999, 341:1420-25.

6. Yates $C$, Amyes $S$ : Extended spectrum $\beta$ lactamases in non typhoidal Salmonella spp. isolated in UK are now a reality: why the late arrival? J Antimicrob Chemother 2005, 56:262-64.
7. Koneman EW, Allen SD, Janda WM, Schereckenberger PC, Winn JWC Color Atlas and Text Book of Diagnostic Microbiology 5th edition. Lippincott, Philadelphia - New York; 1997.

8. Clinical and laboratory standard institute: Performance standards for antimicrobial susceptibility testing; sixteenth informational supplement. Clinical and laboratory standard institute; 2006:26.

9. Hakanen A, Kotilanien P, Jalava J, et al.: Detection of increased fluoroquinolone susceptibility in Salmonella and validation of nalidixic acid screening test. J Clin Microbiol 1999, 37:3572-7.

10. Threlfall EJ, Skinner JA, Graham A, Ward LR, Smith HR: Resistance to ceftriaxone and cefotaxime in non typhoidal Salmonella enterica in England and Wales, 1998-99. J Antimicrob Chemother 2000, 46:847-63.

11. Li WC, Huang FY, Liu CP, Weng LC, Wang NY, Chiu NC, Chiang CS: Ceftriaxone resistance of nontyphoidal Salmonella enterica isolates in Northern Taiwan attributable to production of CTX-M-14 and CMY-2 beta-lactamases. J Clin Microbiol 2005, 43:3237-43.

12. Miriagou V, Tzouvelekis LS, Rossiter S, Tzelepi E, Angulo FJ, Whichard JM: Imipenem resistance in a Salmonella clinical strain due to plasmidmediated class A carbapenemase KPC-2. Antimicrob Agents Chemother 2003, 47:1297-300.

13. Cruchaga S, Echeita A, Aladuena A, Garcia-Pena J, Frias N, Usera MA: Antimicrobial resistance in salmonellae from humans, food and animals in Spain in 1998. J Antimicrob Chemother 2001, 47:315-21.

14. Threlfall EJ, Ward LR, Skinner JA, Rowe B: Increase in multiple antibiotic resistance in nontyphoidal salmonellas from humans in England and Wales: a comparison of data for 1994 and 1996. Microb Drug Resis 1997, 3:263-6.

15. Helms M, Simonsen J, Molbak K: Quinolone resistance is associated with increased risk of invasive illness or death during infection with Salmonella serotype Typhimurium. J Infect Dis 2004, 190:1652-4 
16. Helms M, Vastrup P, Gerner-Smidt P, Mølbak K: Excess mortality associated with antimicrobial drug-resistant Salmonella typhimurium. Emerg Infect Dis 2002, 8:490-5.

17. Kariuki S, Revathi G, Kiiru J, Lowe B, Berkley JA, Hart CA: Decreasing prevalence of antimicrobial resistance in non typhoidal Salmonella isolated from children in a rural district hospital, Kenya. Int J Antimicrob Agents 2006, 28:166-71.

18. Okeke IN, Laxminarayan R, Bhutta ZA, Duse AG, Jenkins P, O'Brien TF, et al.: Antimicrobial resistance in developing countries. Part 1: recent trends and current status. Lancet Infect Dis 2005, 5:481-93.

\section{Pre-publication history}

The pre-publication history for this paper can be accessed here: http://www.biomedcentral.com/1471-2334/10/101/prepub

doi: 10.1186/1471-2334-10-101

Cite this article as: Jabeen et al., Increase in isolation of extended spectrum beta lactamase producing multidrug resistant non typhoidal Salmonellae in Pakistan BMC Infectious Diseases 2010, 10:101

Submit your next manuscript to BioMed Central and take full advantage of:

- Convenient online submission

- Thorough peer review

- No space constraints or color figure charges

- Immediate publication on acceptance

- Inclusion in PubMed, CAS, Scopus and Google Scholar

- Research which is freely available for redistribution

Submit your manuscript at www.biomedcentral.com/submit
C Biomed Central 